\title{
HIV-1 Vif inhibits $G$ to A hypermutations catalyzed by virus-encapsidated APOBEC3G to maintain HIV-1 infectivity
}

\author{
Yudi Wang ${ }^{1}$, Ballington L Kinlock ${ }^{1,2}$, Qiujia Shao ${ }^{1}$, Tiffany M Turner ${ }^{1,2}$ and Bindong Liu ${ }^{1,2^{*}}$
}

\begin{abstract}
Background: HIV-1 viral infectivity factor (Vif) is an essential accessory protein for HIV-1 replication. The predominant function of Vif is to counteract Apolipoprotein B mRNA-editing enzyme-catalytic polypeptide-like 3G (APOBEC3G, A3G), a potent host restriction factor that inhibits HIV-1 replication. Vif mediates the proteasomal degradation of A3G and inhibits A3G translation, thus diminishing the pool of A3G that is available to be packaged into budding virion. Although Vif is robust in degrading A3G, the protection provided against A3G is not absolute. Clinical and laboratory evidence have shown that A3G is not completely excluded from HIV-1 viral particles during HIV-1 replication. It remains unclear why the viral samples are still infectious when A3G has been packaged into the virions.

Results: In this study, we provide evidence that Vif continues to protect HIV-1 from the deleterious effects of A3G, even after packaging of $A 3 G$ has occurred. When equal amounts of $A 3 G$ were packaged into budding virions, the virus expressing functional Vif was more infectious and incurred fewer $\mathrm{G}$ to $\mathrm{A}$ hypermutations in the second round of infection compared to Vif-deficient virus. A Vif mutant with a defect in viral packaging showed a reduced ability to protect the HIV-1 genome from $\mathrm{G}$ to A hypermutations.

Conclusion: Our data suggest that even packaged A3G is still under the tyranny of Vif. Our work brings to light an additional caveat for any therapy that hopes to exploit the Vif-A3G axis. The ideal strategy would not only enhance A3G viral packaging, but also reduce HIV-1 Vif viral encapsidation.
\end{abstract}

Keywords: HIV-1, Vif, APOBEC3G, Hypermutation, Cytidine deaminase

\section{Background}

Apolipoprotein B mRNA-editing enzyme-catalytic polypeptide-like 3G (APOBEC3G, A3G) is a member of the APOBEC3 family of cytidine deaminases, which includes A3A, B, C, D/E and F, with A3G being the most potent against HIV-1 [1]. In the absence of Vif, A3G will encapsidate into HIV-1 virions and induce G to A hypermutations in the newly synthesized viral DNA [2-6]. A3G also exerts inhibitory effects at several other steps of HIV1 replication, such as reverse transcription and viral DNA integration [1].

HIV-1 Vif is a $23 \mathrm{kDa}$ accessory protein of HIV-1. The critical role of Vif in HIV-1 infectivity was observed

\footnotetext{
* Correspondence: bliu@mmc.edu

${ }^{1}$ Center for AIDS Health Disparities Research, 1005 Dr. D. B. Todd Blvd Nashville, Tennessee 37208, USA

${ }^{2}$ Department of Microbiology and Immunology, Meharry Medical College,

1005 Dr. D. B. Todd Blvd, Nashville, Tennessee 37208, USA
}

shortly after the discovery of HIV-1 [7,8]; however, the mechanism by which Vif protects the integrity of HIV-1 took over a decade to decode. Seminal work by Sheehy et al. led to the discovery that the predominant function of Vif is to counteract A3G [9]. The general consensus is that Vif orchestrates proteasomal degradation of A3G, thus preventing its packaging into the budding virion [10-16]. During this process, HIV-1 Vif interacts with Cullin 5, A3G and the newly identified $T$ cell differentiation factor, $\mathrm{CBf} \beta$, to promote formation of the Cullin 5Vif- A3G ubiquitin E3 ligase complex, which marks A3G for proteasomal degradation [16-21]. While Vif-mediated degradation of A3G is the well-recognized mechanism by which Vif rescues HIV-1, it has also been proposed that Vif prevents A3G viral packaging through inhibiting A3G translation [15,22] or another unknown mechanism [23].

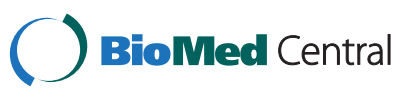

(c) 2014 Wang et al.; licensee BioMed Central Ltd. This is an Open Access article distributed under the terms of the Creative Commons Attribution License (http://creativecommons.org/licenses/by/4.0), which permits unrestricted use, distribution, and reproduction in any medium, provided the original work is properly credited. The Creative Commons Public Domain Dedication waiver (http://creativecommons.org/publicdomain/zero/1.0/) applies to the data made available in this article, unless otherwise stated. 
In addition to inhibiting A3G packaging, it has also been shown that Vif has the capability of directly inhibiting A3G cytidine deaminase activity in a degradationindependent manner. Santa-Marta et al. showed that A3G-induced cytidine deamination is inhibited by the expression of Vif, without the depletion of a deaminase domain, in an Escherichia coli system. Moreover, inhibition of deaminase-mediated bacterial hypermutation is dependent on a single amino acid substitution D128K that renders A3G resistant to Vif inhibition [24]. BritanRosich et al. also showed that Vif is able to inhibit A3G cytidine deaminase activity in vitro [25]. Recently, Feng et al. reported that HIV-1 Vif alters processive singlestranded DNA scanning of A3G in vitro [26]. However, it remains unclear if the inhibitory effect of Vif on A3G cytidine deaminase activity is integral for successful HIV-1 replication following Vif-A3G encapsidation into progeny virions. In this study, we provide evidence that Vif continues to protect HIV-1 from the deleterious effects of A3G even after packaging of A3G has occurred. When equal amounts of A3G were packaged into budding virions with functional or non-functional Vif, the virus expressing functional Vif was more infectious, and fewer $G$ to A hypermutations were generated in the second round of infection compared to virions without functional Vif. Our work indicates that Vif plays an additional role in protecting HIV-1 from A3G after encapsidation. This work sheds light on the fact that any therapy that hopes to exploit the Vif-A3G axis needs to take into account that even though A3G becomes packaged, Vif is still able to maintain the fidelity of the virus.

\section{Results}

Antiviral activity of A3G is less potent against wild-type virus compared to Vif-deficient virus

HIV-1 Vif counteracts A3G antiviral function by mediating its degradation, which leads to the exclusion of A3G from budding viral particles. However, a previous study has shown that residual amounts of A3G still exists in Vif-competent wild-type HIV-1 particles [27]. To study the antiviral function of the residual amount of A3G found in progeny virions, different amount of A3G were cotransfected with HXB2N into 293T cells. Concurrently, A3G was cotransfected with $\mathrm{HXB} 2 \mathrm{~N} \Delta$ Vif into $293 \mathrm{~T}$ cells. Culture supernatants were harvested from the transfected cells $48 \mathrm{~h}$ after transfection. The samples were purified by ultracentrifugation and subjected to MAGI assay and Western blot analysis for measuring viral infectivity and A3G expression levels in progeny virions, respectively. As shown in Figure 1, the amount of A3G in the virion from $20 \mu \mathrm{g}$ A3G/HXB2N sample was more than $4 \mu \mathrm{g}$ A3G/HXB2N $\Delta$ Vif sample (169\% vs $100 \%)$. However, the infectivity of the former virus was much higher than the one of $4 \mu \mathrm{g}$ A3G/HXB2N $\Delta$ Vif sample (Figure 1 lane 5 vs lane 7). These data suggest that wild-type HIV-1 circumvents A3G antiviral function more efficiently than Vif-deficient virus, and thus Vif may have an additional ability to overcome A3G even after A3G has been packaged into viral particles.

\section{HIV-1 Vif inhibits A3G cytidine deaminase activity in both cells and virion}

In our previous report, we demonstrated that a hemagglutinin (HA) tag fused to the $\mathrm{N}$ terminus of lysine-free A3G (HAA3G22K) renders HAA3G22K resistant to Vif-induced degradation [28]. HAA3G22K is equally packaged into wild-type and Vif-deficient HIV-1 virions. Therefore, HAA3G22K is an optimal mutant to characterize the antiviral function of $A 3 G$ in the presence of HIV-1 Vif. To that end, HXB2N, HXB2N $\Delta$ Vif or HXB2B3 was cotransfected into 293T cells with HA A3G22K or A3GD128K. HXB2B3 contains three point mutations in the $\mathrm{C}$-terminal region of Vif as described in the Methods section. Cells and culture supernatants were harvested $48 \mathrm{~h}$ post-transfection, and viral particles were isolated from the culture supernatants by ultracentrifugation. Cells and viral samples were subjected to the A3G cytidine deaminase assay. When Vif (Figure 2B, HXB2N) or VifB3 (Figure 2B, HXB2B3) were co-expressed with HAA3G22K in $293 \mathrm{~T}$ cells, the cytidine deaminase activity of HAA3G22K decreased by $50 \%$ compared to Vif-deficient virus (Figure 2B, HXB2N $\Delta$ Vif). Even though HAA3G22K was packaged equally into the three different virions (Figure 2A), the cytidine deaminase activity of HAA3G22K was lower by 3 - and 2-fold in $\mathrm{HXB} 2 \mathrm{~N}$ and HXB2B3, respectively, compared to HX$\mathrm{B} 2 \mathrm{~N} \Delta$ Vif (Figure 2B). These data suggest that Vif inhibits HAA3G22K cytidine deaminase activity not only in the cells, but also in the virions. When A3GD128K was used instead of HAA3G22K, there was no difference in A3GD128K cytidine deaminase activity among all three viral constructs in both the cells and virions (Figure 2C). As it has been shown that the Vif-A3G interplay is blocked when the 128 lysine residue in A3G is mutated to aspartic acid [29-31], this result (Figure 2C) suggests that the Vif-A3G interaction is essential for Vif to inhibit A3G cytidine deaminase activity.

\section{HIV-1 Vif reduces the $G$ to $A$ hypermutation rate catalyzed by $\mathrm{A} 3 \mathrm{G}$}

With the knowledge that Vif inhibits A3G cytidine deaminase activity in both cells and virions (Figure 2), we set out to determine if Vif interferes with the $G$ to $A$ hypermutation rate catalyzed by $\mathrm{A} 3 \mathrm{G}$. The $\mathrm{HIV}-1$ proviral constructs $\mathrm{HXB} 2 \mathrm{~N}, \mathrm{HXB} 2 \mathrm{~N} \Delta$ Vif or HXB2B3 were cotransfected into 293T cells with A3G, HAA3G22K or A3GD128K. Culture supernatants were harvested $48 \mathrm{~h}$ post-transfection to determine the infectivity of progeny 


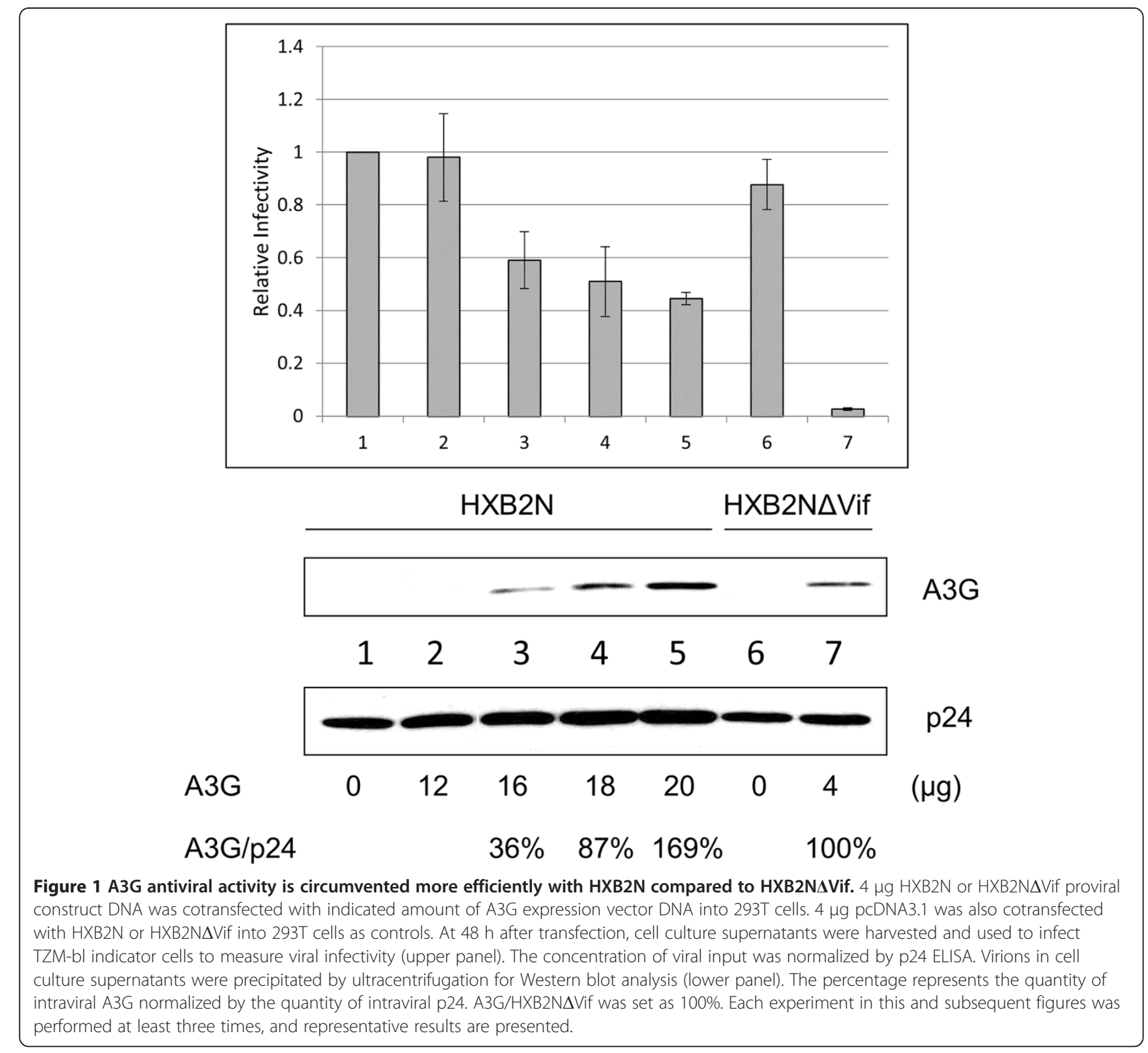

virions and to assay hypermutation levels. As shown in Figure 3A, HXB2B3 retains its capability to degrade A3G as effectively as wildtype HXB2N. Therefore the infectivity HXB2B3/pcDNA3.1 is similar to HXB2B3/A3G (Figure 3B). HAA3G22K was equally packaged into HXB2N, HXB2N $\triangle$ Vif and HXB2B3 (Figure 3A). Although HAA3G22K was equally packaged, the infectivity of HXB2N/HAA3G22K was approximately 10 times higher than that of HXB2N $\triangle$ Vif/HAA3G22K (Figure 3B). The infectivity of HXB2B3/HAA3G22K was in the intermediate range. It is imperative to note that the only difference among these three viruses is the presence of Vif. This finding further illustrates that Vif possesses antiA3G activity even in progeny virions. The $G$ to A hypermutation rate catalyzed by HXB2N/HAA3G22K was significantly lower than that of HXB2N $\triangle$ Vif/HAA3G22K (Figure 3C), while that of HXB2B3 ranged in between those values. This result indicates that Vif reduces the $\mathrm{G}$ to A hypermutation rate catalyzed by A3G even after A3G is packaged into viral particles. Using the A3GD128K mutant instead of HAA3G22K showed that Vif was unable to maintain viral infectivity (Figure $3 \mathrm{~B}$ ), and the rate of $\mathrm{G}$ to A hypermutation (Figure $3 \mathrm{C}$ ) was constant, further indicating that Vif requires interaction with A3G to maintain viral infectivity.

VifB3 mutant encapsidation into HIV particles is defective Compared to wild-type Vif, VifB3 is less efficient in inhibiting A3G cytidine deaminase activity in virions (Figure 2B), reducing $\mathrm{G}$ to $\mathrm{A}$ hypermutations (Figure $3 \mathrm{C}$ ) and retaining 


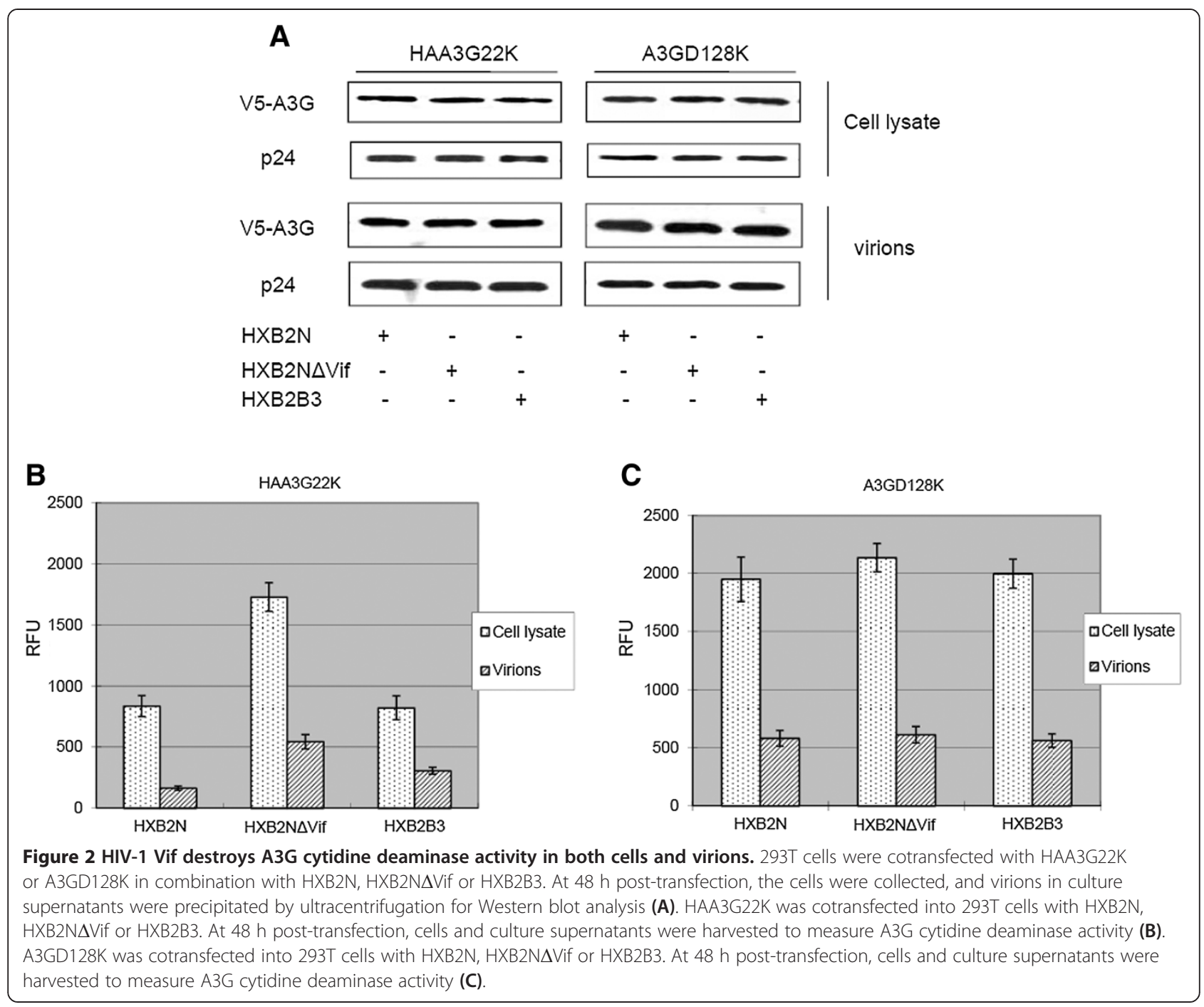

viral infectivity (Figure 3B, HAA3G22K). The viral replication of the HXB2B3 mutant has been reported to be similar to that of wild-type HIV-1 virus in SupT1 cells. However, the HXB2B3 mutant virus shows delayed replication in $\mathrm{H} 9$ cells compared to wild-type virus [32]. Unlike the high levels of A3G found in H9 cells, SupT1 cells express negligible to no A3G. From these observations, we considered that the VifB3 mutant may have a defect in overcoming A3G antiviral activity compared to wild-type Vif. To test this hypothesis, we transfected HXB2N, HXB2N $\triangle$ Vif or HXB2B3 with A3G or HAA3G22K into $293 \mathrm{~T}$ cells. Cells and viral samples were analyzed by Western blot. Both HXB2N and HXB2B3 efficiently induced the degradation of $A 3 G$ in cells and excluded A3G from viral particles (Figure 4A). As expected, HAA3G22K was resistant to HXB2N- and HXB2B3-induced degradation (Figure 4A). Surprisingly, the HXB2B3 virus packaged less Vif compared to wild-type HXB2N virus despite equal expression levels found in the cells (Figure 4A). Similar results were also obtained in the absence of A3G or HAA3G22K, which suggests that VifB3 packaging is independent of A3G and HAA3G22K (Figure 4B). Taken together (Figures 2 and 3), these data show that packaging of Vif is essential for it to inhibit A3G cytidine deaminase activity in virions, reduce the $\mathrm{G}$ to $\mathrm{A}$ hypermutation rate and retain HIV-1 infectivity. Less VifB3 than wild-type Vif was packaged into budding virions, which may explain why the activity of VifB3 always ranged between that of wild-type Vif and no Vif (i.e., Vif-deficient virus) (Figures 2 and 3).

\section{Vif retains infectivity but HIV-1 replication is delayed in HAA3G22K-expressing Jurkat cells}

We have shown that Vif partially retains HIV-1 infectivity even after HAA3G22K was packaged into the budding virion (Figure $3 \mathrm{~B}$ ). To further explore this phenomenon in a $\mathrm{T}$ cell system, we established Jurkat cell lines stably expressing A3G or HAA3G22K. Western blot analysis showed that similar levels of A3G and HAA3G22K were 

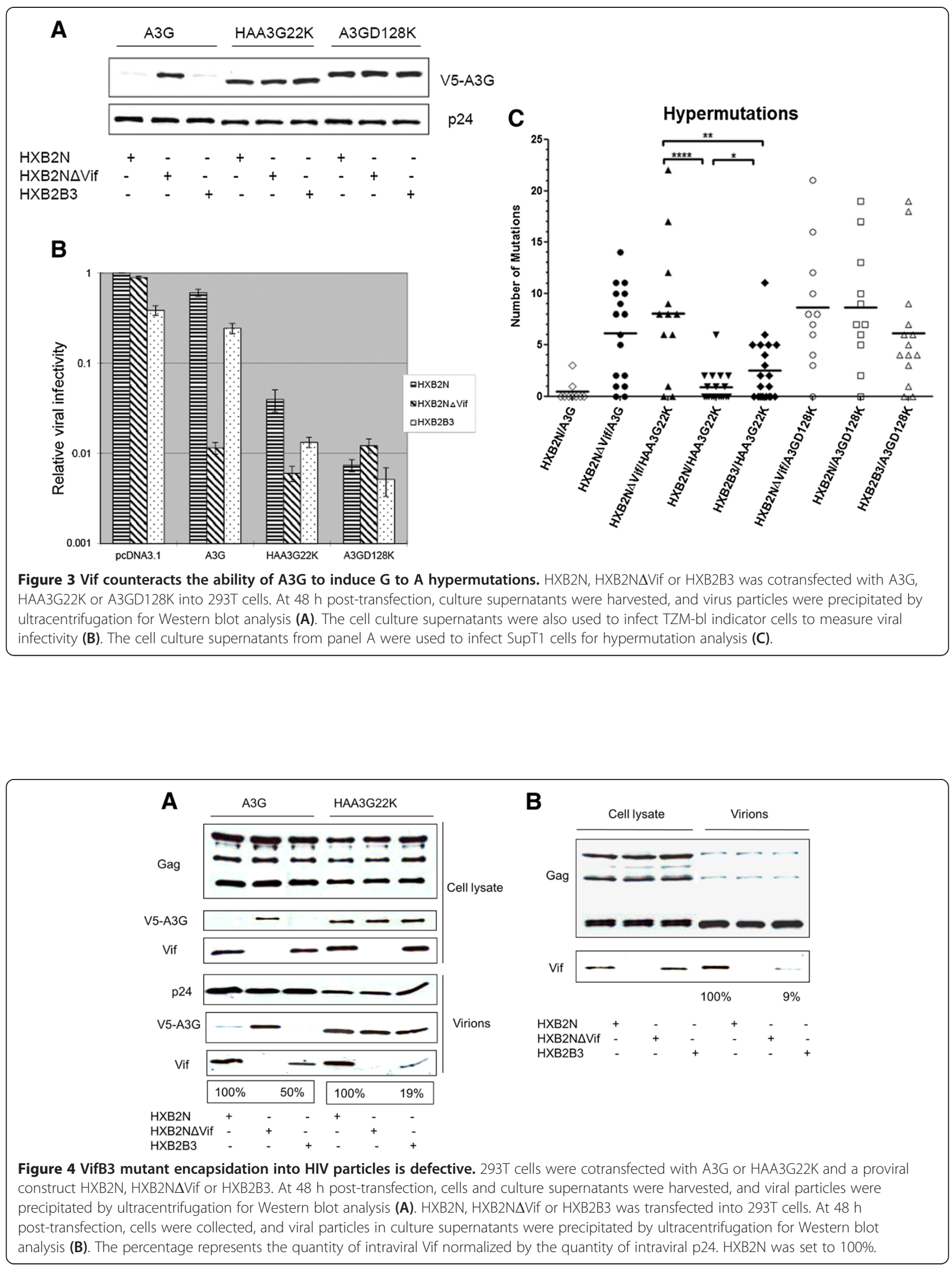
expressed in the selected cell line (Figure 5A). HXB2N, HXB2N $\triangle$ Vif or HXB2B3 virus was used to infect Jurkat, Jurkat/A3G and Jurkat/HAA3G22K cell lines. As expected, all three viruses replicated very well in Jurkat cell line (Figure $5 \mathrm{~B}$ ), while HXB2N $\Delta$ Vif could not replicate in the Jurkat/A3G (Figure 5C) and Jurkat/HAA3G22K (Figure 5D) cell lines. Wild-type HXB2N virus only partially replicated in the Jurkat/HAA3G22K cell line (Figure 5D) compared to its replication in Jurkat (Figure 5B) and Jurkat/A3G (Figure 5C) cell lines. These results indicate that Vif was able to partially maintain HXB2N viral infectivity even after HAA3G22K was packaged into HXB2N virions. In addition, the replication of HXB2B3 was determined not to be robust as HXB2N due to reduced packaging of VifB3.

\section{Discussion}

The discovery of A3G as a potent anti-HIV-1 host restriction factor has fostered interest in blocking the VifA3G interaction and ultimately inhibiting Vif-mediated
A3G degradation as a potential treatment strategy. Vif drastically reduces the cellular pool of A3G and consequently prevents A3G from hitch-hiking a ride with the nucleocapsid of Gag, viral RNA or 7SL RNA into the budding virion, thus preserving HIV-1 infectivity [33]. Several studies have also shown that Vif prevents A3G viral packaging through inhibiting A3G translation [15,22] or other mechanisms [23].

A3G inhibits HIV-1 replication at several steps of the HIV-1 life cycle, such as reverse transcription and viral cDNA integration. However, the hallmark of the potent antiviral phenotype of A3G is found in its ability to induce lethal $\mathrm{G}$ to $\mathrm{A}$ hypermutations in the viral genome. A3G has a strong bias towards catalyzing hypermutations on minus strand DNA 5' CC or 5' CCC. These mutations may lead to the introduction of premature stop codons and/or result in non-infectious or defective proviruses [34]. Although Vif is robust in degrading A3G, thus maintaining viral infectivity, the protection provided against A3G is not absolute. This notion is evident

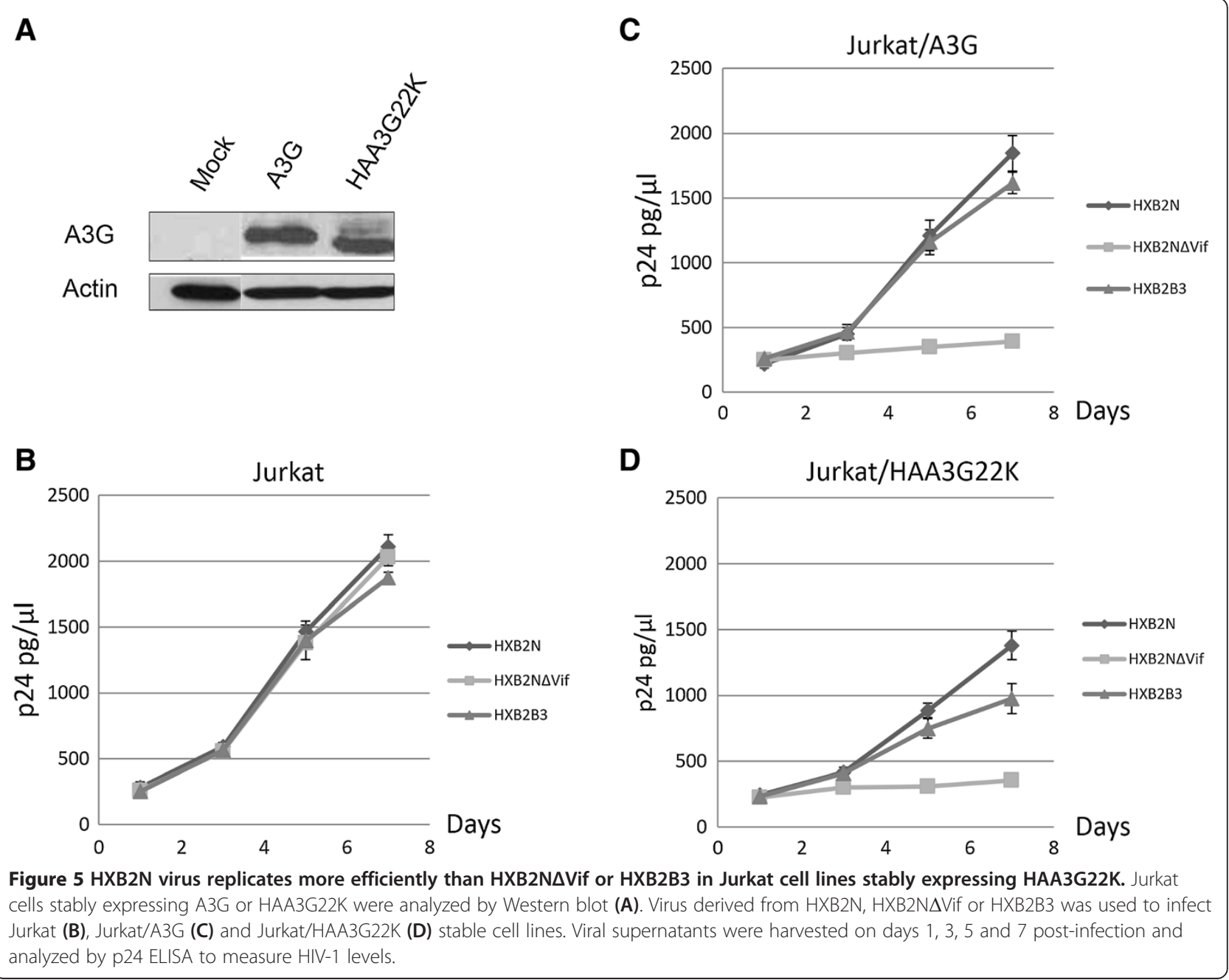


by the $\mathrm{G}$ to $\mathrm{A}$ hypermutations found in genomes of primary viruses isolated from AIDS patients [35-38]. The dominant mutation patterns found among those hypermutations were in the GG to AG context, which suggests that these mutations were induced by A3G. These findings illustrate that $\mathrm{A} 3 \mathrm{G}$ is not completely excluded from HIV-1 viral particles even in the presence of Vif. Observations by Gillick et al. suggest that detectable amounts of A3G are present in wild-type HIV-1 particles produced from $\mathrm{CD} 4^{+} \mathrm{T}$ cells during the course of infection [27]. However, $\mathrm{Xu}$ et al. reported that virion incorporation of approximately seven A3G molecules is sufficient to inhibit the replication of Vif-deficient HIV1 [39]. The obvious discrepancy is that the primary isolates from viral samples were Vif-competent virus, whereas the latter study involved Vif-deficient virus. Taken together, these studies give credence to the argument that Vif may preserve HIV-1 infectivity even after A3G has been packaged into the virion, and our results are consistent with this idea. As shown in Figure 1, even though more A3G was incorporated into Vif-competent HIV (sample 5) compared to Vif-incompetent HIV (sample 7), the infectivity of Vif-competent HIV was much higher than the one of Vif -incompetent HIV. Furthermore, when HAA3G22K, the A3G mutant resistant to Vif-mediated degradation, was used to ensure equal packaging of A3G into both wild-type and Vifdeficient viruses, the infectivity of wild-type virus was 10 -fold greater than that of Vif-deficient virus. This result corresponds to the $\mathrm{G}$ to A hypermutation rate of wild-type virus, which was significantly lower than that of Vif-deficient virus (Figure 3). Ultimately, our results provide evidence that Vif harbors a backup mechanism to counteract A3G antiviral function even after A3G is packaged into the budding virion.

Our lab previously demonstrated that A3G reduces early and late viral reverse transcription products equally in the presence or absence of Vif [28]. This ability of A3G may explain why the viral infectivity of wild-type virus HXB2N in the presence of HAA3G22K was reduced 10fold compared to HXB2N with the pcDNA3.1 control vector in our system (Figure 3B). In the absence of Vif, however, A3G would further diminish the integrity of the viral genome through $G$ to $A$ hypermutations in the newly synthesized viral cDNA, which would not occur in a Vif-proficient virus, as shown in Figure 3C. These data suggest that $G$ to A hypermutations further reduce the infectivity of Vif-deficient HIV-1. A3G cytidine deaminase inactive mutants (such as H257R, C288S and C291S) inhibit HIV-1 replication through reducing viral cDNA production [40]. These mutants do not generate G to A hypermutations [41]. Therefore, we do not expect Vif will have effects on the antiviral function of the intraviral form of these mutants.

\section{Conclusions}

In summary, we used an HIV-1 replication system to show that HIV-1 Vif has alternate functions for counteracting A3G and preserving HIV-1 infectivity. While the initial function of Vif is to prevent A3G viral incorporation, this work confirms that Vif also inhibits the $G$ to A hypermutations catalyzed by A3G. Therefore, any therapy that hopes to exploit the Vif-A3G axis must take into account the ability of Vif to overcome A3G in both the producer cell and the virion. Thus, even though A3G becomes packaged, Vif is still able to maintain the fidelity of the virus. A practical strategy for an antiviral treatment would be not only to increase A3G viral incorporation but also to reduce Vif viral packaging.

\section{Methods}

Plasmids, antibodies and reagents

The proviral DNA constructs of wild-type HIV-1 (HXB2N) and Vif mutant (HXB2N $\Delta$ Vif) as well as VifcMyc expression vectors were provided by Xiao-Fang $\mathrm{Yu}$ (Johns Hopkins University) [11,16]. HXB2B3 contains three point mutations in the C-terminal region of Vif as previously described [42]. The three point mutations were first introduced into a Vif transfer vector, which contained the EcoRI- EcoRI DNA fragment of HXB2N, using the primer GATGGAACAAGCCCCAGGCGACCGCGGGC CACGCAGGGAGCCACACAATGA and QuikChange Lightning Multi Site-Directed Mutagenesis Kit (Agilent Technologies). The DNA fragment containing the three point mutations was then sub-cloned into the HXB2N construct to generate HXB2B3. Wild-type A3G and A3GD128K mutant plasmids were gifts from Yong-Hui Zheng (Michigan State University). The Vif-resistant A3G mutant, HA-A3G20K/R $\Delta 2 \mathrm{~K}$ (hereafter referred to as HAA3G22K), was previously described [28]. The monoclonal anti-V5 antibody was purchased from Invitrogen. The rabbit anti-Vif antibody, rabbit anti-A3G antibody [43] and HIV-1 p24 monoclonal antibody [44] were obtained from NIH-ARRRP.

\section{Cell culture, HIV-1 preparation, HIV-1 purification, DNA transfection, Western blot analysis and viral infectivity (MAGI) assay}

Human embryonic kidney (HEK) 293T cells and TZM-bl cells [45] (NIH-ARRRP) were cultured in DMEM containing $10 \%$ fetal bovine serum (FBS) in $5 \% \mathrm{CO}_{2}$ atmosphere at $37^{\circ} \mathrm{C}$. The culture supernatant was harvested $48 \mathrm{~h}$ post-transfection for the MAGI assay and viral preparation. HIV-1 virions were prepared from the cell culture supernatant and separated from cellular debris by centrifugation at $1,000 \times g$ for $15 \mathrm{~min}$ and filtered through a $0.2 \mu \mathrm{m}$ pore size membrane. Virus particles were concentrated by ultracentrifugation at $100,000 \times g$ for $2 \mathrm{~h}$ on a $20 \%$ sucrose cushion. Transfections were 
performed using polyethylenimine (PEI). Western blot analysis and the MAGI assay were carried out as previously described $[28,46]$.

\section{Hypermutation assay}

293T cells were cotransfected with HIV-1 expression vectors and A3G expression vectors as indicated. Culture supernatants were collected $48 \mathrm{~h}$ post-transfection and treated with DNase $\mathrm{I}(20 \mathrm{U} / \mathrm{ml})$ at $37^{\circ} \mathrm{C}$ for $1 \mathrm{~h}$. SupT1 cells $\left(1 \times 10^{6}\right)$ were spin-infected with DNase Itreated HIV-1 (150 $\mathrm{ng} \mathrm{p} 24^{\mathrm{Gag}}$ equivalent) at $2000 \times g$ for $2 \mathrm{~h}$ at room temperature. The cells were then washed with fresh medium and cultured at $37^{\circ} \mathrm{C}, 5 \% \mathrm{CO}_{2}$ atmosphere. Cells were harvested $12 \mathrm{~h}$ post-infection. DNA was isolated using a DNeasy Blood and Tissue DNA isolation kit (QIAGEN). A 650-bp DNA fragment covering a portion of $n e f$, U3, and R of HIV-1 was amplified with Taq DNA polymerase (Invitrogen) using the primers HIV-1-F (5'-AGGCAGCTGTAGATATTAGCCAC) and HIV-1-R (5' -GTATGAGGGATCTCTAGCTACCA). The PCR products were cloned into the TOPO TA-cloning vector pCR2.1 (Invitrogen). The clones were sequenced, and the sequencing results were analyzed using the CLC Main Workbench software. Statistical analysis was performed using the GraphPad Prism 5 software.

\section{A3G cytidine deaminase assay}

The FRET-based cytidine deaminase assay was performed based on a modified protocol adapted from a previously described method [47]. 293T cells were cotransfected with the indicated HIV-1 expression vector and the indicated A3G expression vector. At $48 \mathrm{~h}$ post-transfection, cells and supernatant were harvested, and the virus was concentrated from the supernatant by ultracentrifugation. NP40 buffer (0.626\% NP40, $10 \mathrm{mM}$ Tris acetate $\mathrm{pH} 7.5,50 \mathrm{mM}$ potassium acetate, $10 \mathrm{mM} \mathrm{NaCl}$ ) was used to lyse both cell and viral samples. The cell or viral lysate $(15-20 \mu \mathrm{l})$ was mixed with $70 \mu \mathrm{l}$ master mix containing 20 pmol Taqman positive control probe (56-FAM/ TTATTATTCCCATTTGATT/-36TAMSp), 1.0 unit uracil DNA glycosylase (NEB), $50 \mathrm{mM}$ Tris ( $\mathrm{pH} 7.4$ ), $10 \mathrm{mM}$ EDTA, $250 \mu \mathrm{g} / \mathrm{ml}$ RNase A (Qiagen) and $1 \mu \mathrm{M}$ fluorescence. Cell and viral lysate samples were incubated at $37^{\circ} \mathrm{C}$ for $3 \mathrm{~h}$ or overnight, respectively. Thereafter, $4 \mu \mathrm{l}$ of $4 \mathrm{M} \mathrm{NaOH}$ was added to the reaction and incubated for $30 \mathrm{~min}$ at $37^{\circ} \mathrm{C}$. The reaction was then neutralized by adding $4 \mu \mathrm{l}$ of $4 \mathrm{M} \mathrm{HCl}$ and $36 \mu$ of $2 \mathrm{M}$ Tris ( $\mathrm{pH}$ 7.9). The samples were cooled to $4^{\circ} \mathrm{C}$, and fluorescence was measured using a MyiQ ICycler (Bio-Rad). To measure the background of each sample, the Taqman negative control probe (56-FAM/TTATTATTGGGATTTGATT/36-TAMSp) was used. The cytidine deaminase activity was calculated as a function of the activity generated from the positive probe minus the activity generated from the negative probe.

\section{Jurkat A3G and Jurkat HAA3G22K stably expressing cell lines and HIV-1 replication}

To establish stable cell lines, Jurkat cells $\left(1 \times 10^{6}\right)$ were washed twice with PBS and pulsed with the A3G or HAA3G22K plasmid (3 $\mu$ g each) suspended in $10 \mu \mathrm{l}$ Buffer $\mathrm{R}$ using the Invitrogen Neon Transfection System (pulse voltage $1425 \mathrm{~V}$, pulse width $10 \mathrm{~ms}$ and pulse number 3). After electroporation, the cells were cultured in RPMI with 10\% FBS for two days. G418 $(1 \mathrm{mg} / \mathrm{ml}$, Invitrogen) was added to the culture on day 3 for neomycin resistance selection. Single clones were selected using the limited dilution method. The APOBEC expression of the selected cell lines was monitored by Western blot. HXB2N, HXB2N $\triangle$ Vif and HXB2B3 proviral constructs were transfected into $293 \mathrm{~T}$ cells to produce the corresponding viruses. Jurkat, Jurkat/A3G and Jurkat/ HAA3G22K cells $\left(6 \times 10^{5}\right)$ were spin-infected with $150 \mathrm{ng}$ of p24 HXB2N, HXB2N $\Delta$ Vif or HXB2B3 virus. After extensive washes with RPMI, the infected Jurkat cell lines were cultured at $37^{\circ} \mathrm{C}$ in a $5 \% \mathrm{CO}_{2}$ incubator, and the supernatant $(200 \mu \mathrm{l})$ was harvested on days 1,3 , 5 and 7 post-infection. An equal volume of fresh culture media was added to the culture to maintain a constant culture volume after each harvest. HIV-1 levels were assayed using a p24 ELISA kit (AIDS and Cancer Virus Program, National Cancer Institute, Frederick, MD).

\section{Competing interests}

The authors declare that they have no competing interests.

\section{Authors' contributions}

YW, BLK and QS performed experiments. YW, BLK, QS, TMT and BL contributed to the experimental design and result discussion. YW, BLK, QS and BL wrote the manuscript. All authors read and approved the final manuscript.

\section{Acknowledgements}

We thank X-F Yu (Johns Hopkins University) and Y-H Zheng (Michigan State University) for reagents. The following reagents were obtained through the $\mathrm{NIH}$ AIDS Research and Reference Reagent Program, Division of AIDS, NIAID, NIH: HIV-1HXB2 Vif antiserum; TZM-bl from J C. Kappes, X Wu and Tranzyme Inc; HIV-1 p24 monoclonal antibody from Dr. Bruce Chesebro and Kathy Wehrly; Anti-ApoC17 from Dr. Klaus Strebel.

This work was partially supported by NIH grants SC1GM089269, G12MD007586, U54MD007593, P30Al054999 and UL1TR000445 to BL. BLK and TMT are supported by NIH training grant T32HL007737 and T32Al007281 respectively.

Received: 19 March 2014 Accepted: 23 September 2014

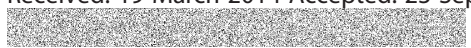

\section{References}

1. Malim MH: APOBEC proteins and intrinsic resistance to HIV-1 infection. Philos Trans R Soc Lond B Biol Sci 2009, 364:675-687.

2. Zhang H, Yang B, Pomerantz RJ, Zhang C, Arunachalam SC, Gao L: The cytidine deaminase CEM15 induces hypermutation in newly synthesized HIV-1 DNA. Nature 2003, 424:94-98. 
3. Mariani R, Chen D, Schrofelbauer B, Navarro F, Konig R, Bollman B, Munk C, Nymark-McMahon H, Landau NR: Species-Specific Exclusion of APOBEC3G from HIV-1 Virions by Vif. Cell 2003, 114:21-31.

4. Mangeat B, Turelli P, Caron G, Friedli M, Perrin L, Trono D: Broad antiretroviral defence by human APOBEC3G through lethal editing of nascent reverse transcripts. Nature 2003, 424:99-103.

5. Lecossier D, Bouchonnet F, Clavel F, Hance AJ: Hypermutation of HIV-1 DNA in the absence of the Vif protein. Science 2003, 300:1112.

6. Harris RS, Bishop KN, Sheehy AM, Craig HM, Petersen-Mahrt SK, Watt IN, Neuberger MS, Malim MH: DNA deamination mediates innate immunity to retroviral infection. Cell 2003, 113:803-809.

7. Fisher AG, Ensoli B, Ivanoff L, Chamberlain M, Petteway S, Ratner L Gallo RC, Wong-Staal F: The sor gene of HIV-1 is required for efficient virus transmission in vitro. Science 1987, 237:888-893.

8. Strebel K, Daugherty D, Clouse K, Cohen D, Folks T, Martin MA: The HIV 'A' (sor) gene product is essential for virus infectivity. Nature 1987, 328:728-730.

9. Sheehy AM, Gaddis NC, Choi JD, Malim MH: Isolation of a human gene that inhibits HIV-1 infection and is suppressed by the viral Vif protein Nature 2002, 418:646-650.

10. Conticello SG, Harris RS, Neuberger MS: The Vif protein of HIV triggers degradation of the human antiretroviral DNA deaminase APOBEC3G. Curr Biol 2003, 13:2009-2013.

11. Liu B, Yu X, Luo K, Yu Y, Yu XF: Influence of primate lentiviral Vif and proteasome inhibitors on human immunodeficiency virus type 1 virion packaging of APOBEC3G. J Virol 2004, 78:2072-2081.

12. Marin M, Rose KM, Kozak SL, Kabat D: HIV-1 Vif protein binds the editing enzyme APOBEC3G and induces its degradation. Nat Med 2003, 9:1398-1403.

13. Mehle A, Strack B, Ancuta P, Zhang C, McPike M, Gabuzda D: Vif overcomes the innate antiviral activity of APOBEC $3 G$ by promoting its degradation in the ubiquitin-proteasome pathway. J Biol Chem 2004, 279:7792-7798.

14. Sheehy AM, Gaddis NC, Malim MH: The antiretroviral enzyme APOBEC3G is degraded by the proteasome in response to HIV-1 Vif. Nat Med 2003, 9:1404-1407.

15. Stopak K, de Noronha C, Yonemoto W, Greene WC: HIV-1 Vif blocks the antiviral activity of APOBEC3G by impairing both its translation and intracellular stability. Mol Cell 2003, 12:591-601.

16. Yu X, Yu Y, Liu B, Luo K, Kong W, Mao P, Yu XF: Induction of APOBEC3G ubiquitination and degradation by an HIV-1 Vif-Cul5-SCF complex. Science 2003, 302:1056-1060.

17. Mehle A, Goncalves J, Santa-Marta M, McPike M, Gabuzda D: Phosphorylation of a novel SOCS-box regulates assembly of the HIV-1 Vif-Cul5 complex that promotes APOBEC3G degradation. Genes Dev 2004, 18:2861-2866.

18. Yu Y, Xiao Z, Ehrlich ES, Yu X, Yu XF: Selective assembly of HIV-1 Vif-Cul5ElonginB-ElonginC E3 ubiquitin ligase complex through a novel SOCS box and upstream cysteines. Genes Dev 2004, 18:2867-2872.

19. Kobayashi M, Takaori-Kondo A, Miyauchi Y, Iwai K, Uchiyama T: Ubiquitination of APOBEC3G by an HIV-1 Vif-Cullin5-Elongin B-Elongin C complex is essential for Vif function. J Biol Chem 2005, 280:18573-18578.

20. Luo K, Xiao Z, Ehrlich E, Yu Y, Liu B, Zheng S, Yu XF: Primate lentiviral virion infectivity factors are substrate receptors that assemble with cullin 5-E3 ligase through a HCCH motif to suppress APOBEC3G. Proc Natl Acad Sci US A 2005, 102:11444-11449.

21. Stanley BJ, Ehrlich ES, Short L, Yu Y, Xiao Z, Yu XF, Xiong Y: Structural insight into the human immunodeficiency virus Vif SOCS box and its role in human E3 ubiquitin ligase assembly. J Virol 2008, 82:8656-8663.

22. Mercenne G, Bernacchi S, Richer D, Bec G, Henriet S, Paillart JC, Marquet R: HIV-1 Vif binds to APOBEC3G mRNA and inhibits its translation. Nucleic Acids Res 2010, 38:633-646

23. Kao S, Khan MA, Miyagi E, Plishka R, Buckler-White A, Strebel K: The Human Immunodeficiency Virus Type 1 Vif Protein Reduces Intracellular Expression and Inhibits Packaging of APOBEC3G (CEM15), a Cellular Inhibitor of Virus Infectivity. J Virol 2003, 77:11398-11407.

24. Santa-Marta M, da Silva FA, Fonseca AM, Goncalves J: HIV-1 Vif can directly inhibit apolipoprotein B mRNA-editing enzyme catalytic polypeptide-like 3G-mediated cytidine deamination by using a single amino acid interaction and without protein degradation. J Biol Chem 2005, 280:8765-8775.

25. Britan-Rosich E, Nowarski R, Kotler M: Multifaceted counter-APOBEC3G mechanisms employed by HIV-1 Vif. J Mol Biol 2011, 410:1065-1076.
26. Feng Y, Love RP, Chelico L: HIV-1 Vif alters processive single-stranded DNA scanning of the retroviral restriction factor APOBEC3G. J Biol Chem 2013, 288:6083-6094.

27. Gillick K, Pollpeter D, Phalora P, Kim EY, Wolinsky SM, Malim MH: Suppression of HIV-1 Infection by APOBEC3 Proteins in Primary Human CD4+ T Cells Is Associated with Inhibition of Processive Reverse Transcription as Well as Excessive Cytidine Deamination. J Virol 2013, 87:1508-1517.

28. Wang Y, Shao Q, Yu X, Kong W, Hildreth JE, Liu B: N-terminal hemagglutinin tag renders lysine-deficient APOBEC3G resistant to HIV-1 Vif-induced degradation by reduced polyubiquitination. J Virol 2011, 85:4510-4519.

29. Bogerd HP, Doehle BP, Wiegand HL, Cullen BR: A single amino acid difference in the host APOBEC3G protein controls the primate species specificity of HIV type 1 virion infectivity factor. Proc Natl Acad Sci U S A 2004, 101:3770-3774.

30. Mangeat $B$, Turelli $P$, Liao S, Trono D: A single amino acid determinant governs the species-specific sensitivity of APOBEC3G to Vif action. J Biol Chem 2004, 279:14481-14483.

31. Schrofelbauer B, Chen D, Landau NR: A single amino acid of APOBEC3G controls its species-specific interaction with virion infectivity factor (Vif) Proc Natl Acad Sci U S A 2004, 101:3927-3932.

32. Bouyac M, Courcoul M, Bertoia G, Baudat Y, Gabuzda D, Blanc D, Chazal N, Boulanger P, Sire J, Vigne R, Spire B: Human immunodeficiency virus type 1 Vif protein binds to the Pr55Gag precursor. J Virol 1997, 71:9358-9365.

33. Strebel K, Khan MA: APOBEC3G encapsidation into HIV-1 virions: which RNA is it? Retrovirology 2008, 5:55

34. Vartanian JP, Meyerhans A, Asjo B, Wain-Hobson S: Selection, recombination, and $\mathrm{G}-\mathrm{-}$ A hypermutation of human immunodeficiency virus type 1 genomes. J Virol 1991, 65:1779-1788.

35. Janini M, Rogers M, Birx DR, McCutchan FE: Human immunodeficiency virus type 1 DNA sequences genetically damaged by hypermutation are often abundant in patient peripheral blood mononuclear cells and may be generated during near-simultaneous infection and activation of CD4(+) T cells. J Virol 2001, 75:7973-7986.

36. Kieffer TL, Kwon P, Nettles RE, Han Y, Ray SC, Siliciano RF: G- > A hypermutation in protease and reverse transcriptase regions of human immunodeficiency virus type 1 residing in resting CD4+ T cells in vivo. J Virol 2005, 79:1975-1980.

37. Kijak GH, Janini LM, Tovanabutra S, Sanders-Buell E, Arroyo MA, Robb ML, Michael NL, Birx DL, McCutchan FE: Variable contexts and levels of hypermutation in HIV-1 proviral genomes recovered from primary peripheral blood mononuclear cells. Virology 2008, 376:101-111.

38. Piantadosi A, Humes D, Chohan B, McClelland RS, Overbaugh J: Analysis of the percentage of human immunodeficiency virus type 1 sequences that are hypermutated and markers of disease progression in a longitudinal cohort, including one individual with a partially defective Vif. J Virol 2009, 83:7805-7814.

39. Xu H, Chertova E, Chen J, Ott DE, Roser JD, Hu WS, Pathak VK: Stoichiometry of the antiviral protein APOBEC3G in HIV-1 virions. Virology 2007, 360:247-256

40. Holmes RK, Koning FA, Bishop KN, Malim MH: APOBEC3F can inhibit the accumulation of HIV-1 reverse transcription products in the absence of hypermutation. Comparisons with APOBEC3G. J Biol Chem 2007, 282:2587-2595.

41. Newman EN, Holmes RK, Craig HM, Klein KC, Lingappa JR, Malim MH, Sheehy AM: Antiviral function of APOBEC3G can be dissociated from cytidine deaminase activity. Curr Biol 2005, 15:166-170.

42. Goncalves J, Shi B, Yang X, Gabuzda D: Biological activity of human immunodeficiency virus type 1 Vif requires membrane targeting by C-terminal basic domains. J Virol 1995, 69:7196-7204.

43. Kao S, Miyagi E, Khan MA, Takeuchi H, Opi S, Goila-Gaur R, Strebel K: Production of infectious human immunodeficiency virus type 1 does not require depletion of $A P O B E C 3 G$ from virus-producing cells. Retrovirology 2004, 1:27.

44. Chesebro B, Wehrly K, Nishio J, Perryman S: Macrophage-tropic human immunodeficiency virus isolates from different patients exhibit unusual V3 envelope sequence homogeneity in comparison with T-cell-tropic isolates: definition of critical amino acids involved in cell tropism. J Virol 1992, 66:6547-6554.

45. Platt EJ, Wehrly K, Kuhmann SE, Chesebro B, Kabat D: Effects of CCR5 and CD4 cell surface concentrations on infections by macrophagetropic 
isolates of human immunodeficiency virus type 1. J Virol 1998, 72:2855-2864.

46. Shao Q, Wang Y, Hildreth JE, Liu B: Polyubiquitination of APOBEC3G is essential for its degradation by HIV-1 Vif. J Virol 2010, 84:4840-4844.

47. Thielen BK, Klein KC, Walker LW, Rieck M, Buckner JH, Tomblingson GW, Lingappa JR: T cells contain an RNase-insensitive inhibitor of APOBEC3G deaminase activity. PLoS Pathog 2007, 3:1320-1334.

doi:10.1186/s12977-014-0089-5

Cite this article as: Wang et al: HIV-1 Vif inhibits $G$ to A hypermutations catalyzed by virus-encapsidated APOBEC3G to maintain HIV-1 infectivity. Retrovirology 2014 11:89.

\section{Submit your next manuscript to BioMed Central and take full advantage of:}

- Convenient online submission

- Thorough peer review

- No space constraints or color figure charges

- Immediate publication on acceptance

- Inclusion in PubMed, CAS, Scopus and Google Scholar

- Research which is freely available for redistribution 